\title{
Morphologically tunable MOF nanosheets in mixed matrix membranes for $\mathrm{CO}_{2}$ separation
}

\section{Supporting Information}

Jing Deng ${ }^{\dagger 1}$, Zhongde Dai ${ }^{11}$, Jingwei Hou ${ }^{2}$ and Liyuan Deng ${ }^{* 1}$

AUTHOR ADDRESS

${ }^{1}$ Department of Chemical Engineering, Norwegian University of Science and Technology Trondheim, 7491, Norway.

Email: deng@nt.ntnu.no

${ }^{2}$ School of Chemical Engineering, Faculty of Engineering, Architecture and Information Technology, the University of Queensland, Brisbane, Queensland, Australia

$\dagger$ These authors contributed equally 


\section{Table of contents}

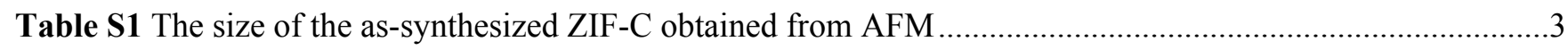

Figure S1 SEM images of A) ZIF-C 30-70, B) ZIF-C 72 and C) ZIF-C 85-124 ...........................................

Figure S2 The $\mathrm{N}_{2}$ adsorption experiment results of ZIF-C prepared in PVA solution ........................................

Table S2 The $\mathrm{N}_{2}$ adsorption experiment results of ZIF-C prepared in PVA solution .........................................4

Figure S3 XPS C 1s, Zn 2p, N 1s and O 1s spectra of ZIF-C 30-70 and benchmark ZIF-L................................5

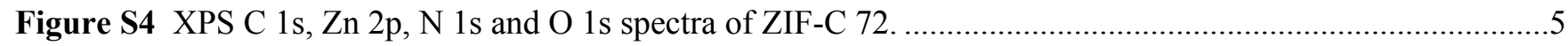

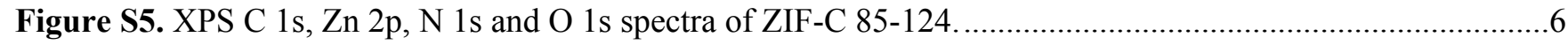

Figure S6 MMMs morphology with A) 0\%, B) 5\%, C) 10\% and D) 20\% ZIF-C 72 ...................................6

Figure S7 MMMs morphology with A) 0\%, B) 5\%, C) 10\% and D) 20\% ZIF-C 30-70 ...............................7

Figure S8 FTIR spectra of MMMs containing A) ZIF-C 30-70, B) ZIF-C 72 and C) ZIF-C 85-124 with a

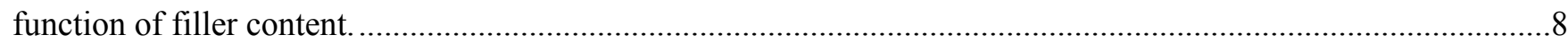

Figure S9 TGA curves of MMMs containing A) ZIF-C 30-70 and B) ZIF-C 72 with a function of filler content. .8 Figure S10 XRD results of MMMs containing A) ZIF-C 30-70 and B) ZIF-C 72 with different content. .............9

Figure S11 (A) $\mathrm{CO}_{2}$ permeability and (B) $\mathrm{CO}_{2} / \mathrm{N}_{2}$ selectivity of Pebax + ZIF-C 30-70 MMMs as a function of $\mathrm{RH}$

Figure S12 (A) $\mathrm{CO}_{2}$ permeability and (B) $\mathrm{CO}_{2} / \mathrm{N}_{2}$ selectivity of Pebax + ZIF-C 72 MMMs as a function of RH. .9 
Table S1 The size of the as-synthesized ZIF-C obtained from AFM

\begin{tabular}{cccc}
\hline MOFs & Length $(\mu \mathrm{m})^{\mathrm{a}}$ & ${\text { Width }(\mu \mathrm{m})^{\mathrm{a}}}$ & ${\text { Thickness }(\mathrm{nm})^{\mathrm{b}}}^{\mathrm{a}}$ \\
ZIF-C 30-70 & $5.0 \pm 0.59$ & $2.0 \pm 0.27$ & $70 \pm 10$ \\
ZIF-C 72 & $4.3 \pm 0.29$ & $1.4 \pm 0.18$ & $120 \pm 10$ \\
ZIF-C 85-124 & $4.1 \pm 0.51$ & $1.1 \pm 0.14$ & $170 \pm 7$ \\
Leaf-like ZIF-C & 5.0 & 2.0 & 150 \\
\hline
\end{tabular}

a: measured from SEM analysis; b: measured from AFM analysis

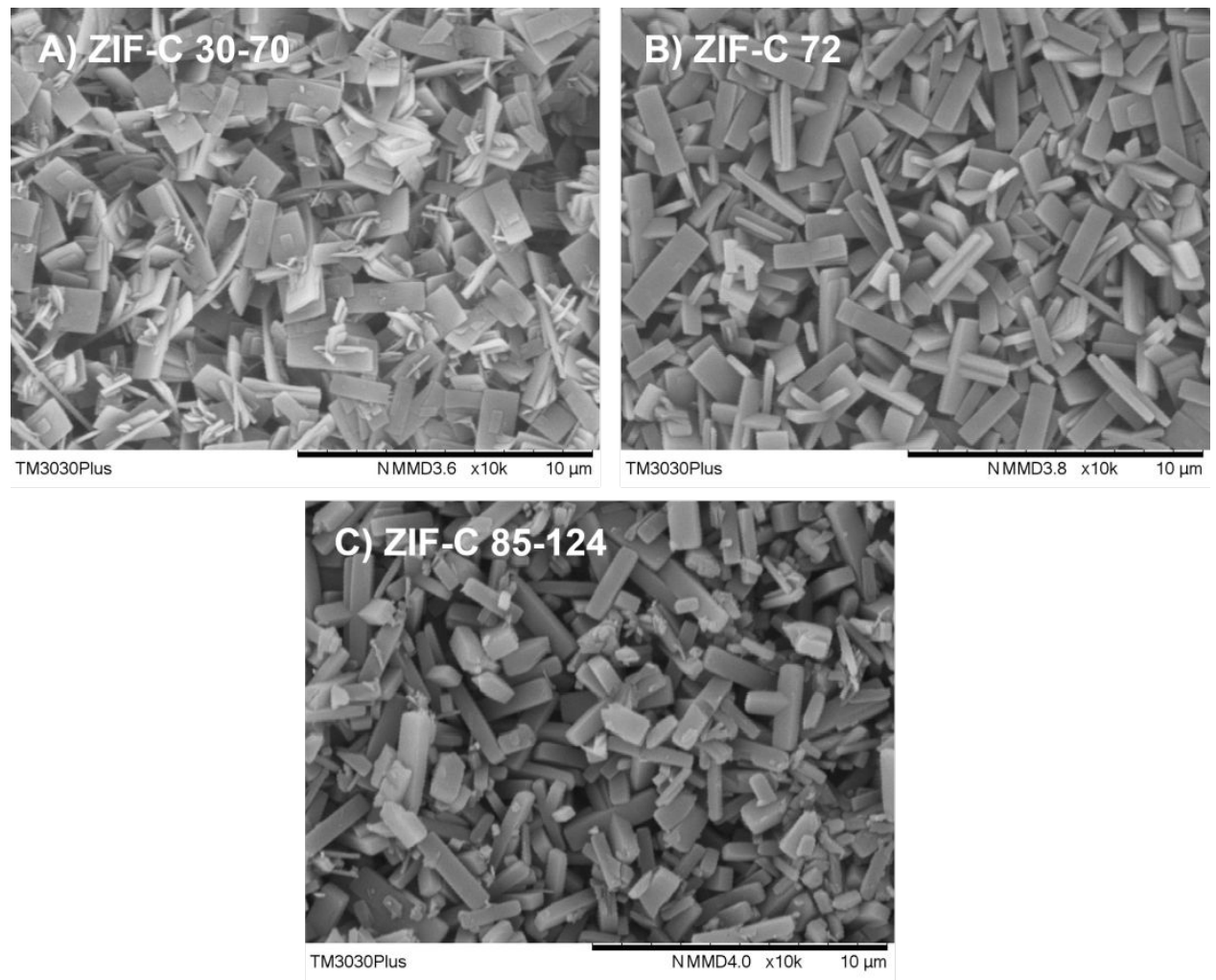

Figure S1 SEM images of A) ZIF-C 30-70, B) ZIF-C 72 and C) ZIF-C 85-124. 
A)

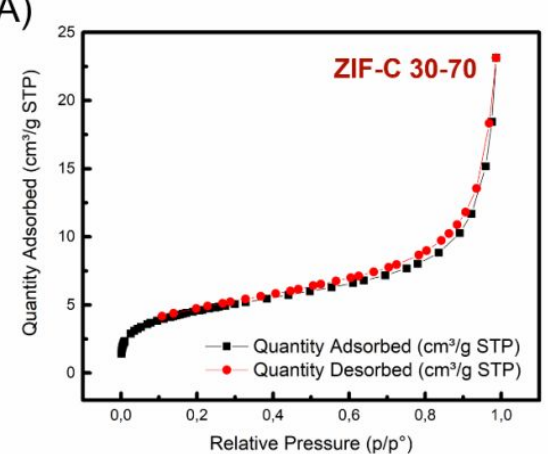

C)

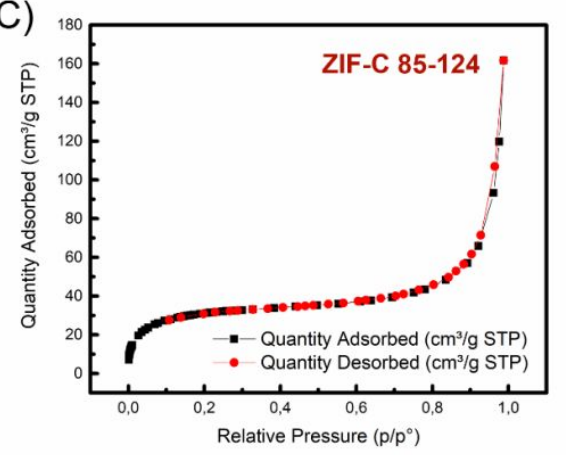

B)
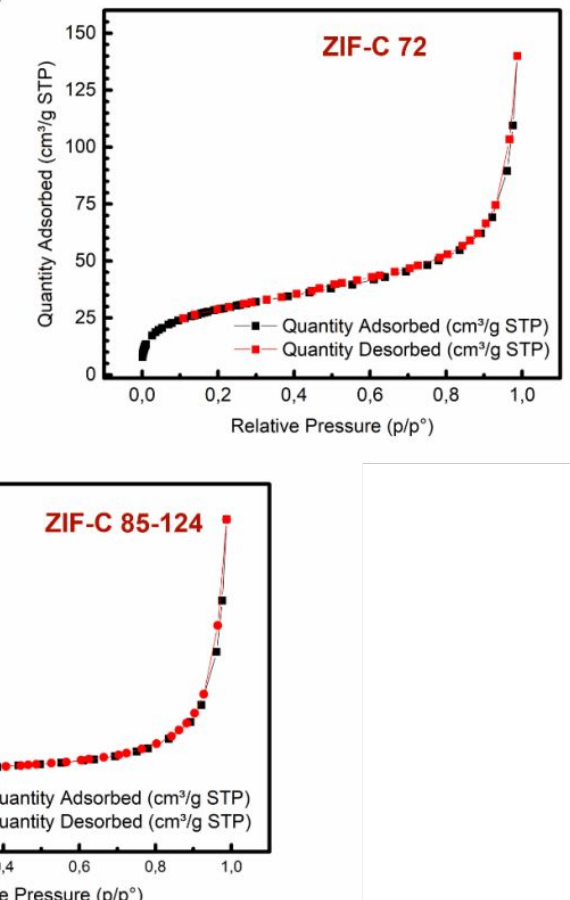

Figure S2 The $\mathrm{N}_{2}$ adsorption experiment results of ZIF-C prepared in PVA solution

Table S2 The $\mathrm{N}_{2}$ adsorption experiment results of ZIF-C prepared in PVA solution

\begin{tabular}{cc}
\hline Fillers & $\mathbf{S}_{\mathbf{B E T}}\left(\mathbf{m}^{\mathbf{2}} \mathbf{g}^{-1}\right)$ \\
\hline ZIF-C 30-70 & $16.0627 \pm 0.1166$ \\
\hline ZIF-C 72 & $101.9866 \pm 0.8517$ \\
\hline ZIF-C 85-124 & $103.7662 \pm 1.4549$ \\
\hline
\end{tabular}



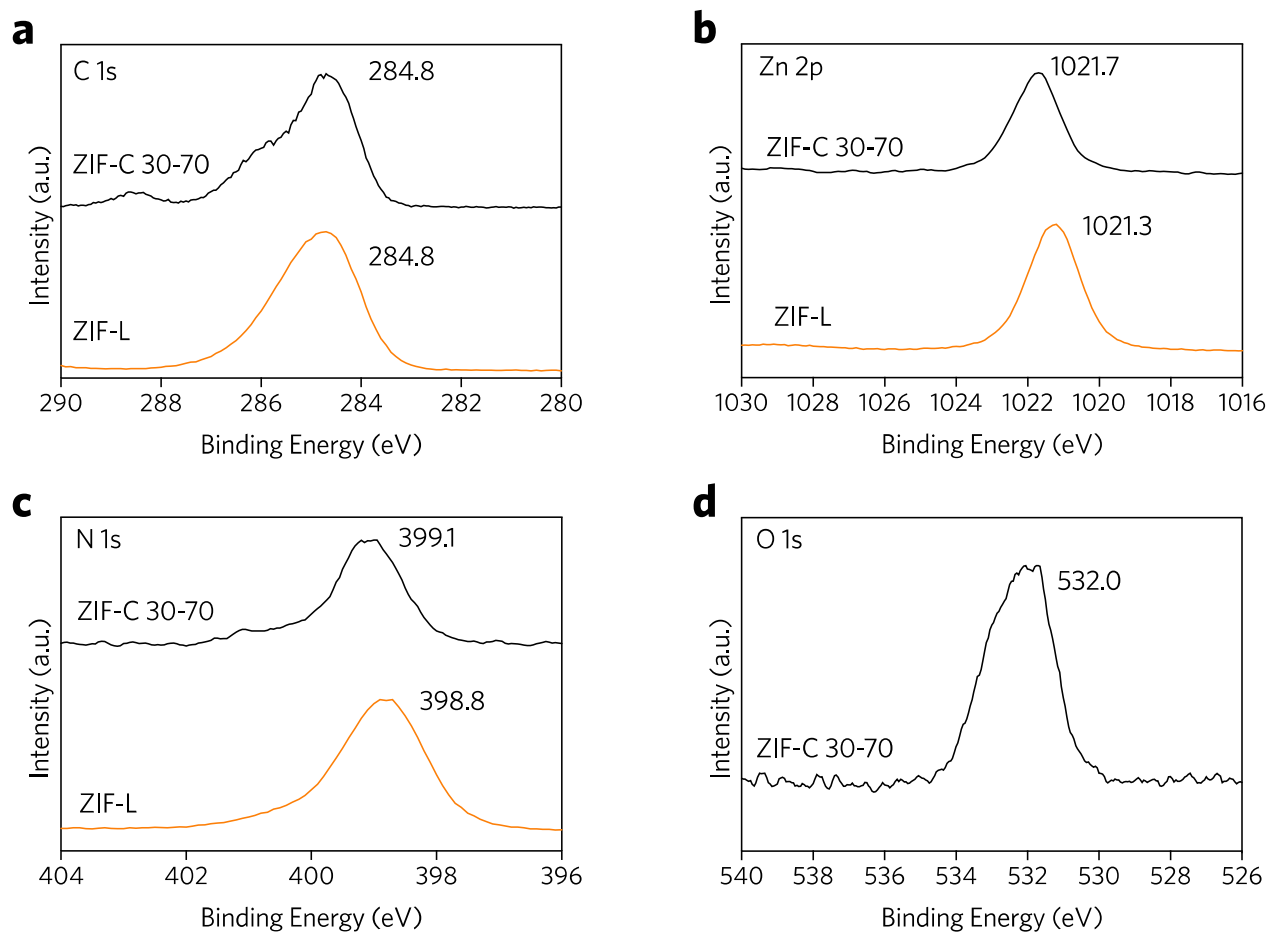

Figure S3 XPS C 1s, Zn 2p, N 1s and O 1s spectra of ZIF-C 30-70 and benchmark ZIF-L.
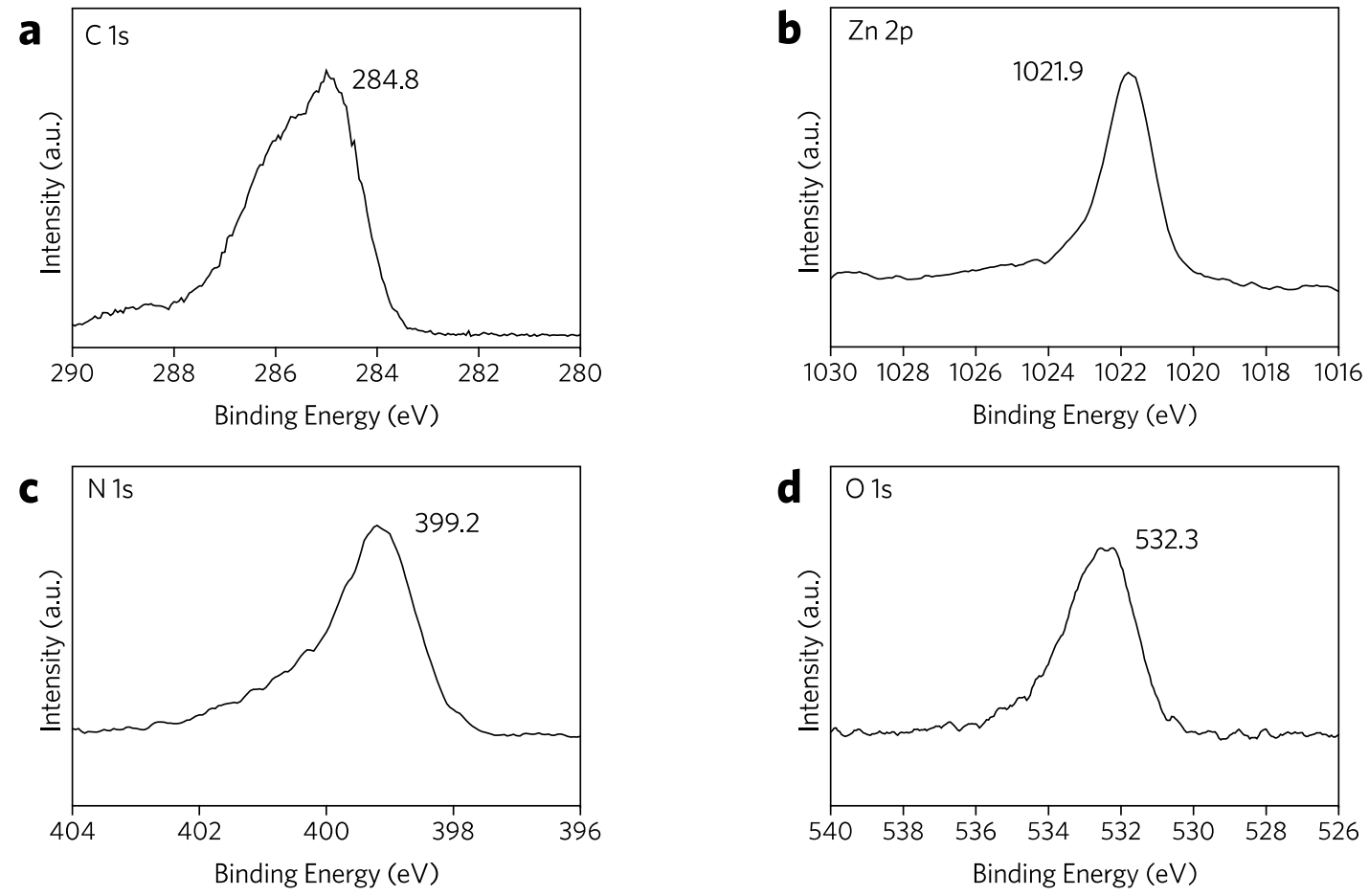

Figure S4 XPS C 1s, Zn 2p, N 1s and O 1s spectra of ZIF-C 72. 

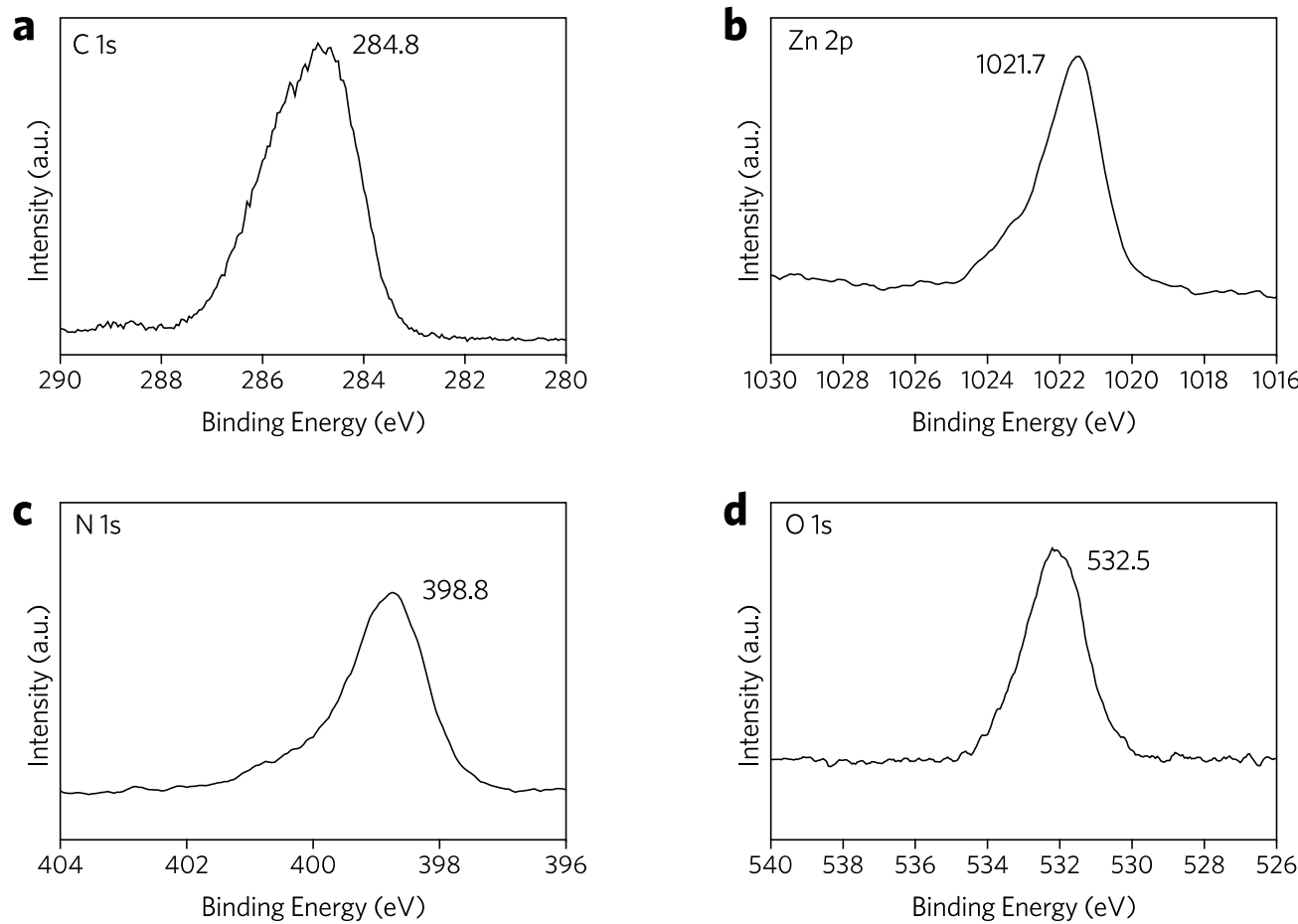

Figure S5. XPS C 1s, Zn 2p, N 1s and O 1s spectra of ZIF-C 85-124.
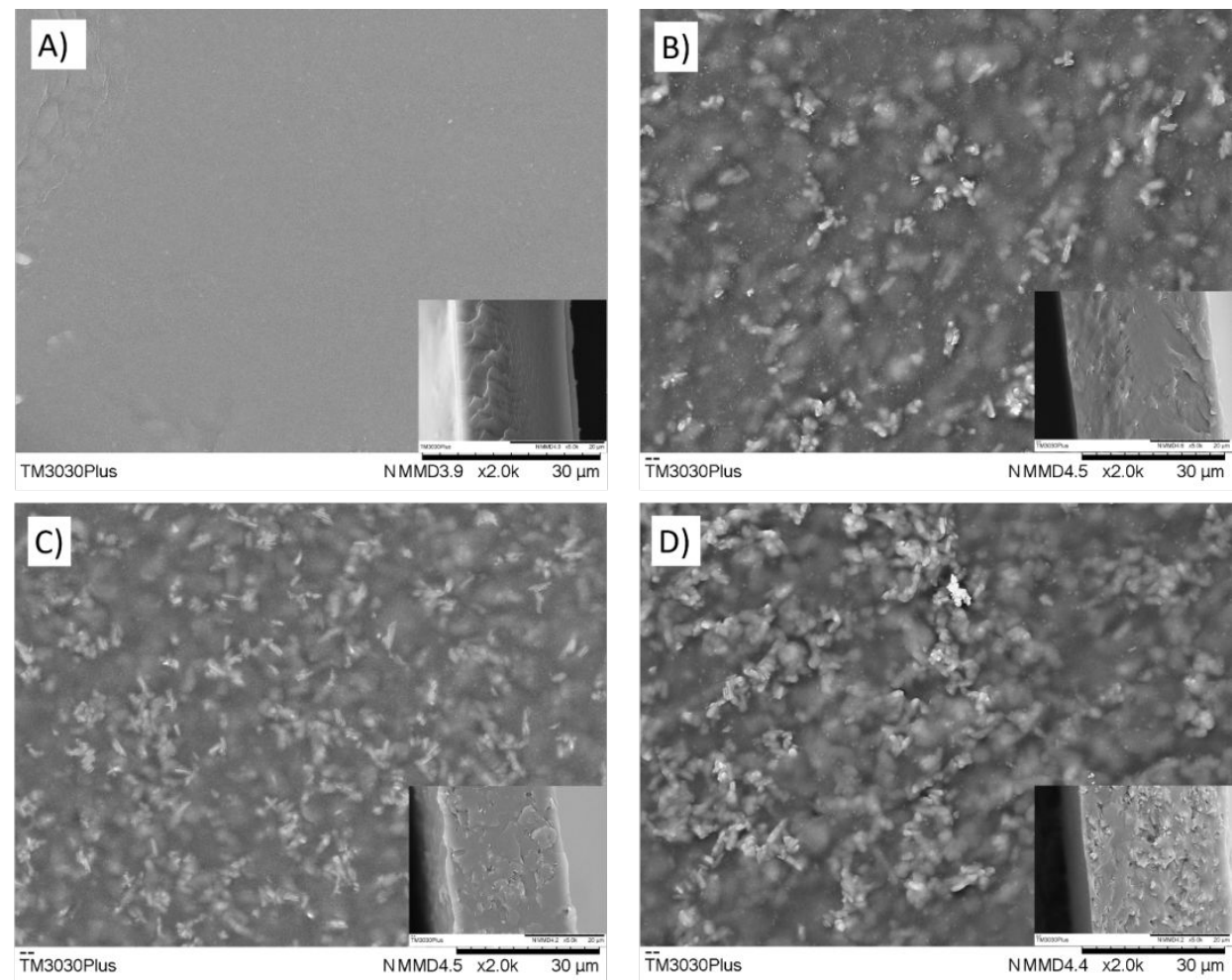

Figure S6 MMMs morphology with A) 0\%, B) 5\%, C) 10\% and D) 20\% ZIF-C 72. 


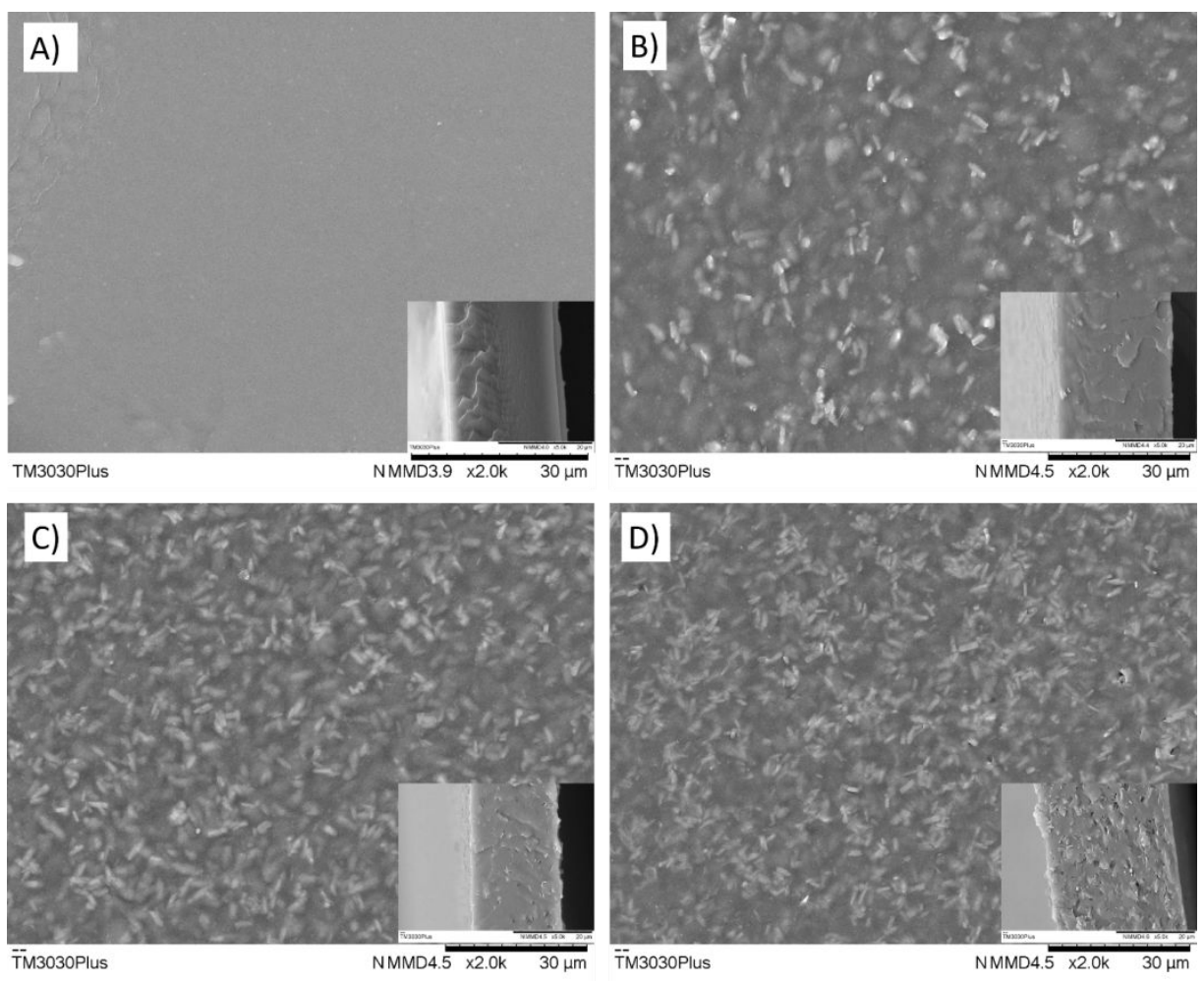

Figure S7 MMMs morphology with A) 0\%, B) 5\%, C) 10\% and D) 20\% ZIF-C 30-70 
A)

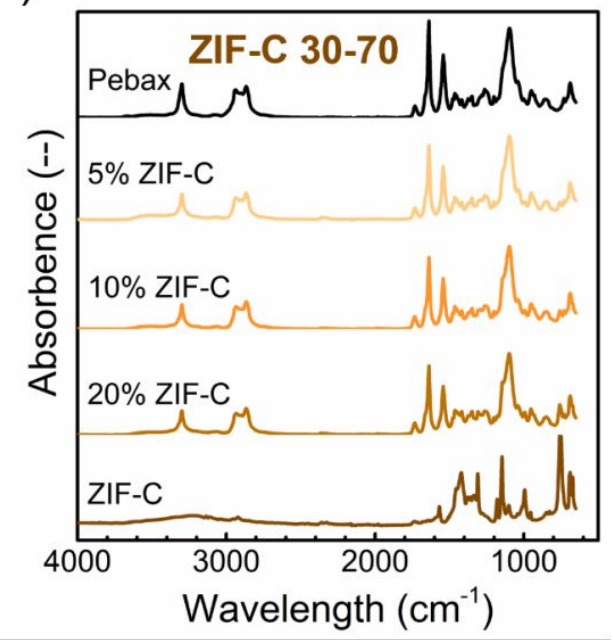

B)

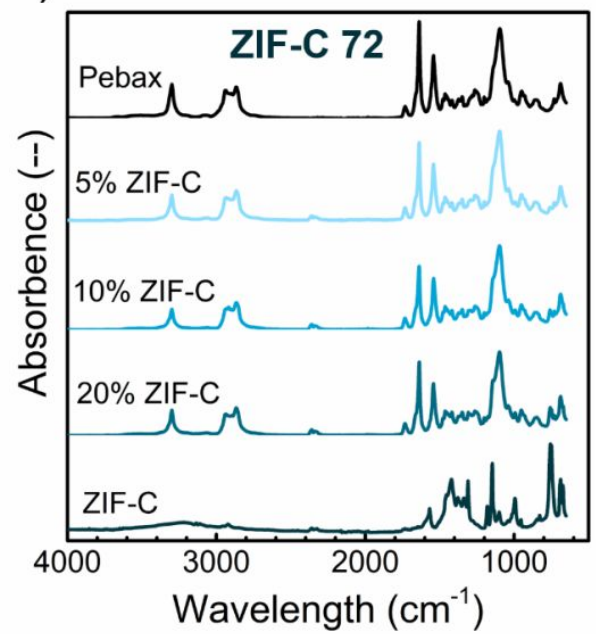

C)

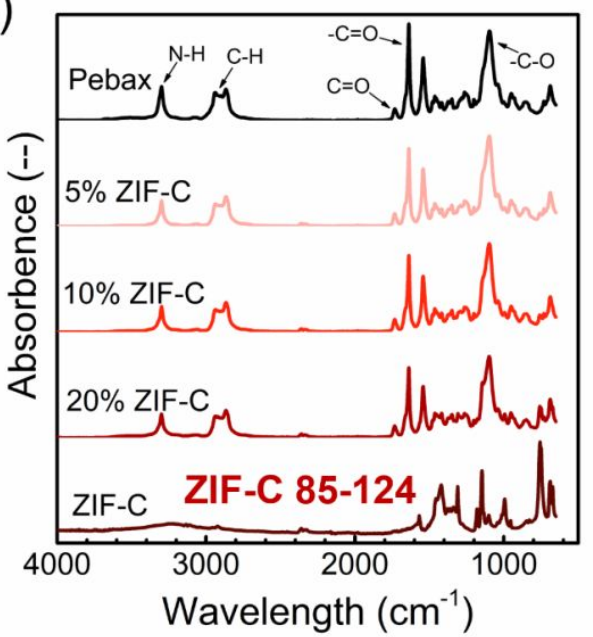

Figure S8 FTIR spectra of MMMs containing A) ZIF-C 30-70, B) ZIF-C 72 and C) ZIF-C 85-124 with a function of filler content.

A)

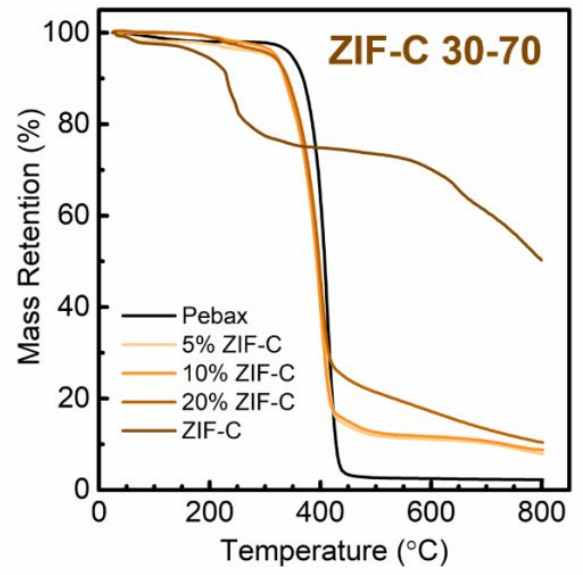

B)

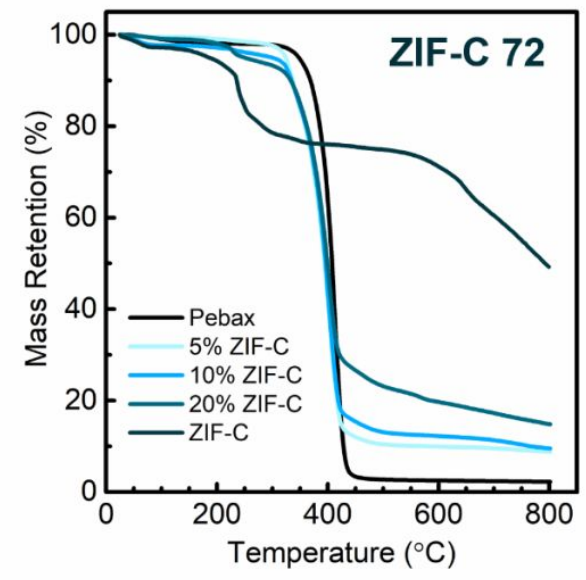

Figure S9 TGA curves of MMMs containing A) ZIF-C 30-70 and B) ZIF-C 72 with a function of filler content. 
A)

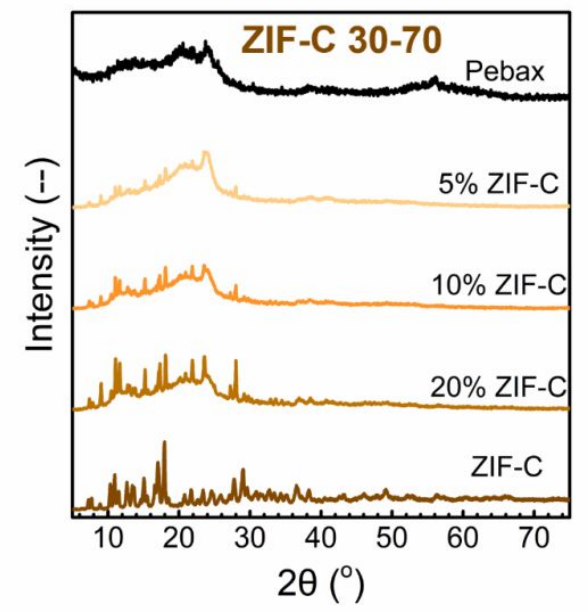

B)

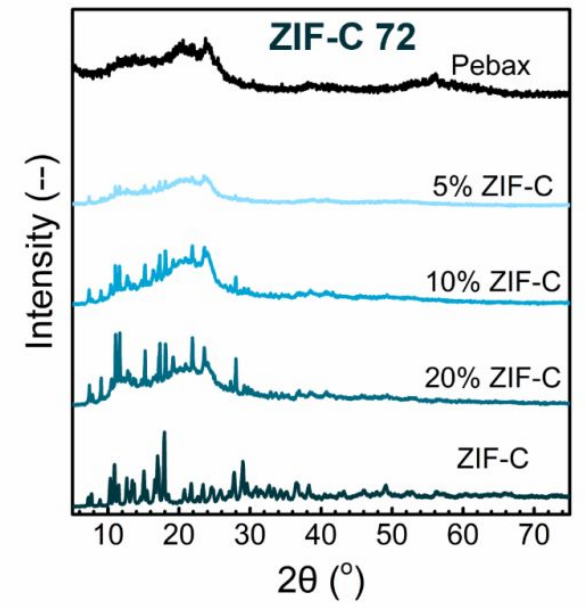

Figure S10 XRD results of MMMs containing A) ZIF-C 30-70 and B) ZIF-C 72 with different content.
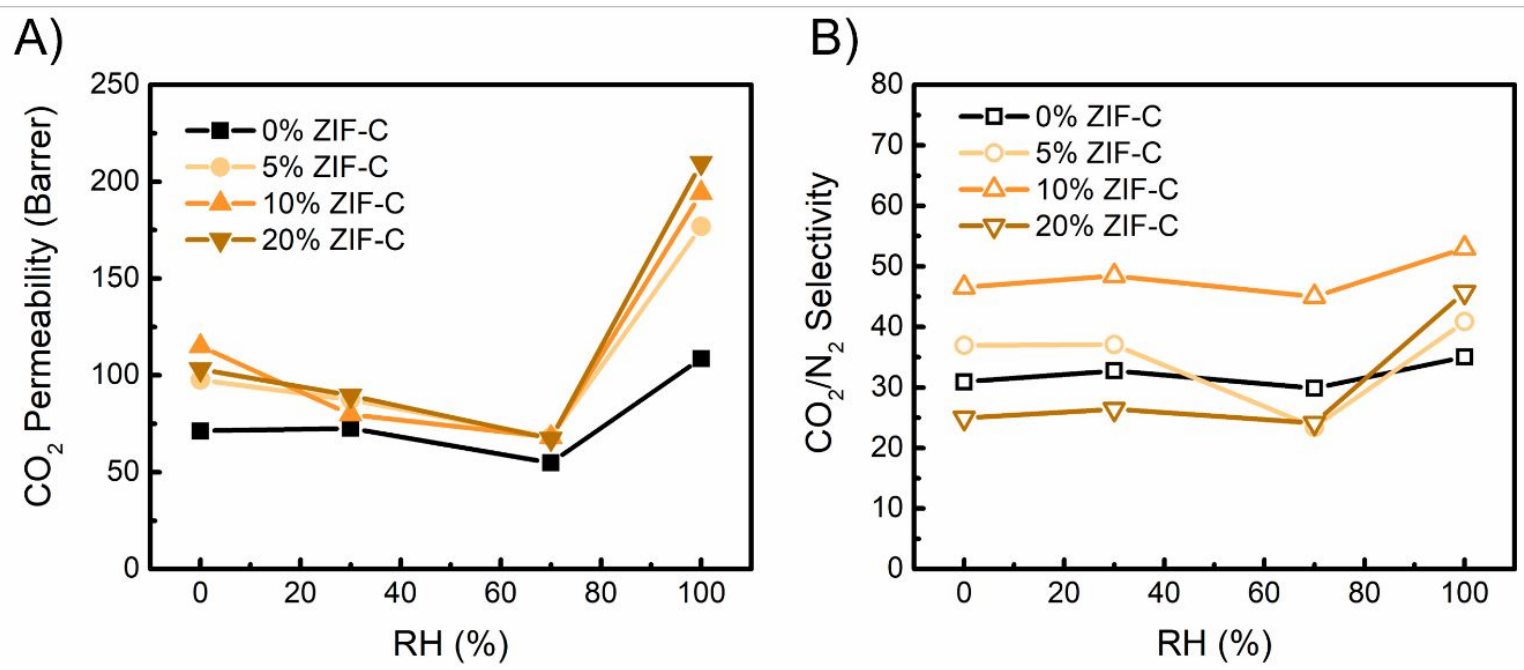

Figure S11 (A) $\mathrm{CO}_{2}$ permeability and (B) $\mathrm{CO}_{2} / \mathrm{N}_{2}$ selectivity of Pebax + ZIF-C 30-70 MMMs as a function of RH.

A)

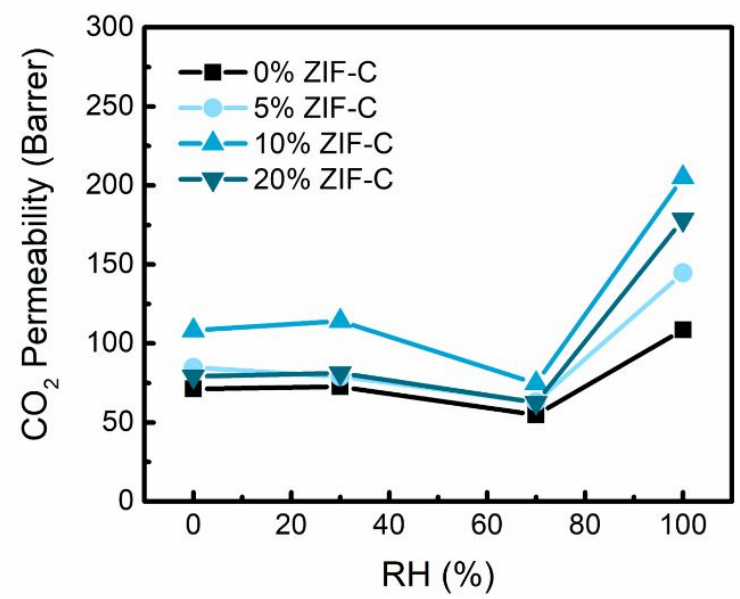

B)

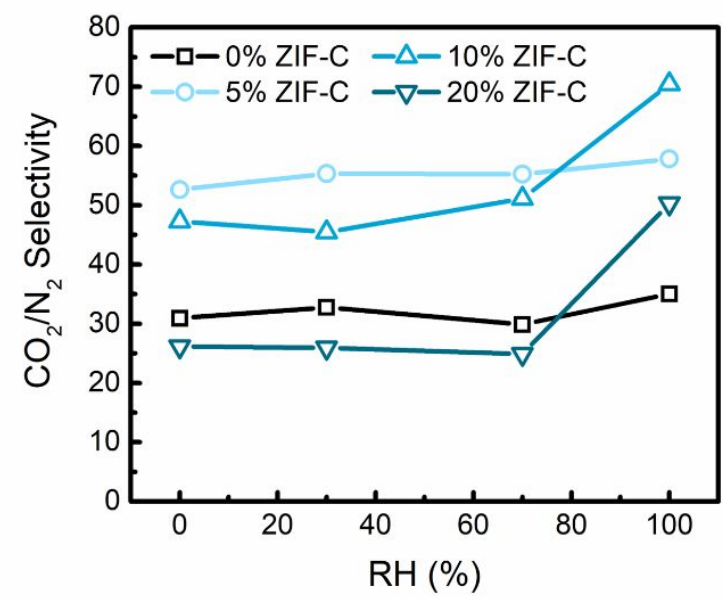

Figure S12 (A) $\mathrm{CO}_{2}$ permeability and (B) $\mathrm{CO}_{2} / \mathrm{N}_{2}$ selectivity of Pebax + ZIF-C $72 \mathrm{MMMs}$ as a function of RH. 


\section{REFERENCE}

1. Chen, R.; Yao, J.; Gu, Q.; Smeets, S.; Baerlocher, C.; Gu, H.; Zhu, D.; Morris, W.; Yaghi, O. M.; Wang, H., A two-dimensional zeolitic imidazolate framework with a cushion-shaped cavity for $\mathrm{CO}_{2}$ adsorption. Chem. Commun. 2013, 49, (82), 9500-2. 